\title{
RÉALISATION D'UN APPAREIL D'ANALYSE THERMIQUE A COMPENSATION PAR DÉRIVATION DE LA TEMPÉRATURE
}

\author{
J. CHICOIS et M. THEOLIER
}

Laboratoire de Métallurgie, Mécanique, INSA Lyon, France

(Reçu le 27 septembre 1972, révisé le 24 janvier 1973)

\begin{abstract}
Résumé. - Description d'un enthalpimètre à compensation par dérivation de la température. Il réunit les caractéristiques de l'analyse thermique dérivée (un seul échantillon employé et grandes vitesses d'évolution) et de l'analyse calorimétrique à compensation (directement quantitative). Discussion des performances de cet appareil et illustration par quelques exemples.
\end{abstract}

Abstract. Description of a derivative temperature compensating calorimeter. The derivative thermal analysis results (using one sample with high speed cycling) and the compensating analytical calorimeter (directly quantitative) results are combined in this apparatus.

Discussion of the performance of this apparatus is illustrated with some examples.

1. Introduction. - La technique la plus utilisée en analyse thermique reste l'analyse thermique différentielle (ATD) [1].

Ce procédé, de mise en œuvre simple, peut devenir quantitatif après un étalonnage long et délicat. Il ne peut s'appliquer, avec une grande sensibilité, que pour des chauffages très lents [2].

L'analyse thermique différentielle à puissance compensée est directement quantitative [3], mais elle nécessite l'emploi d'un témoin et d'asservissements en température très compliqués.

Nous avons construit un appareil d'analyse thermique à compensation plus simple que l'ATD à puissance compensée, utilisant un seul échantillon et directement quantitatif. Cet appareil a fait l'objet d'un brevet d'invention $\mathrm{n}^{0} 72.10 .417$, à la demande de l'ANVAR.

2. Principe général. - La puissance $P$ nécessaire au chauffage d'un échantillon placé dans des conditions adiabatiques idéales peut s'écrire :

$$
P=K m C_{\theta} V_{\theta}
$$

$K=$ constante dépendant des unités utilisées,

$m=$ masse de l'échantillon,

$\theta=$ température à l'instant considéré,

$C_{\theta}=$ chaleur massique à la température $\theta$,

$V_{\theta}=$ vitesse de chauffage à la température $\theta$.

Si la vitesse de chauffage est maintenue constante, l'expression (1) devient

$$
P=K_{1} C_{\theta} \text {. }
$$

Dans ce cas, la puissance nécessaire au chauffage de l'échantillon est directement proportionnelle à sa chaleur massique.

Dans le cas où l'échantillon présente une transformation physicochimique, si la vitesse de chauffage est maintenue constante durant toute cette transformation, la puissance nécessaire pour chauffer cet échantillon présentera une variation égale à la puissance dégagée par la transformation.

La figure 1 représente le cas d'une transformation endothermique. Elle nécessite donc plus de puissance pour maintenir la vitesse de chauffage constante. Cette transformation a entraîné une variation de chaleur massique $\Delta C$.

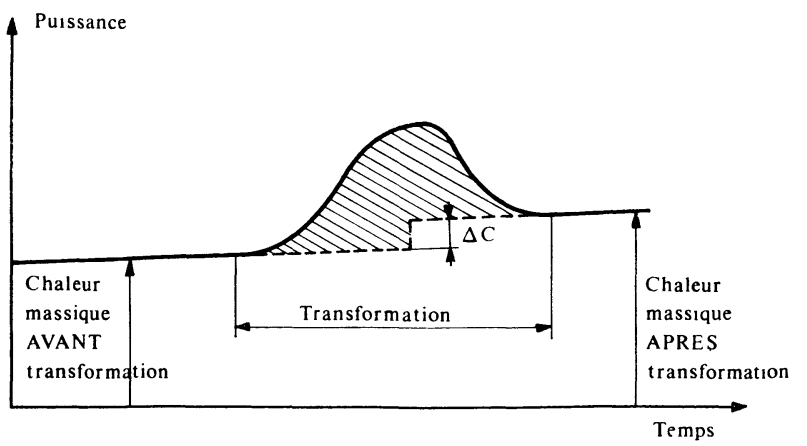

Fig. 1. - Puissance nécessaire au chauffage d'un échantillon présentant une transformation endothermique en fonction de la température.

Certaines règles [4] permettent d'interpoler la ligne de base. L'aire du pic ainsi déterminé (surface hachurée) 
est directement proportionnelle à l'énergie dégagée par la réaction.

Dans le cas de transformations irréversibles, n'entraînant pas de variation de chaleur massique, il suffit d'effectuer un deuxième chauffage dans les mêmes conditions pour obtenir avec précision la ligne de base.

En conclusion, si l'on introduit un élément chauffant à l'intérieur d'un échantillon placé dans des conditions adiabatiques, la puissance fournie à cet élément chauffant pour maintenir la vitesse de chauffage de l'échantillon constante est proportionnelle à la chaleur massique et aux transformations éventuelles de l'échantillon.

3. Schéma de l'installation (Fig. 2). - Un échantillon est placé à l'intérieur d'un four sous vide qui constitue l'enceinte adiabatique.

- Un microfour, formé d'une résistance chauffante, est placé à l'intérieur de l'échantillon pour assurer son chauffage. On peut considérer que l'énergie dégagée par ce microfour est intégralement absorbée par l'échantillon.

- Un thermocouple différentiel permet de mesurer l'écart de température entre l'échantillon et l'enceinte adiabatique. Cet écart est maintenu le plus faible possible par action sur l'élément chauffant du four au moyen d'une commande de puissance.

-- Un thermocouple mesure la température de l'échantillon, un dérivateur électronique détermine sa vitesse de chauffage. Après amplification, cette vitesse de chauffage est comparée à une consigne fixe, Une régulation agissant sur le microfour permet de main- tenir cette vitesse de chauffage égale à la valeur imposée par la consigne.

- Un servomultiplicateur fait le produit du courant traversant le microfour par la tension à ces bornes.

- Un enregistreur suit l'évolution de la puissance fournie au microfour en fonction du temps lorsque l'on maintient constante la vitesse de chauffage de l'échantillon placé dans des conditions adiabatiques.

4. Etude du dérivateur capacitif. - Le thermocouple donne une tension qui est fonction de la température de l'échantillon. Un dérivateur électronique détermine une tension proportionnelle à la vitesse de chauffage.

Le dérivateur doit être suffisamment sensible et stable pour détecter des variations de température très faibles. Le dérivateur à capacité, présenté par Séfram [5] comme application de son amplispot, a servi de base à notre étude.

4.1 Principe du dérivateur a capacité (Fig. 3). - A un instant donné, le courant $i$ circulant dans le circuit est donné par la relation

$$
i=C \frac{\mathrm{d}}{V_{\mathrm{AB}}}
$$

$V_{\mathrm{AB}}=$ tension aux bornes de la capacité;

$C \quad=$ valeur de la capacité.

Si la résistance de l'amplificateur est négligeable, la tension aux bornes de la capacité est égale à la tension $e \mathrm{du}$ thermocouple.

La valeur du courant $i$ qui circule dans le circuit

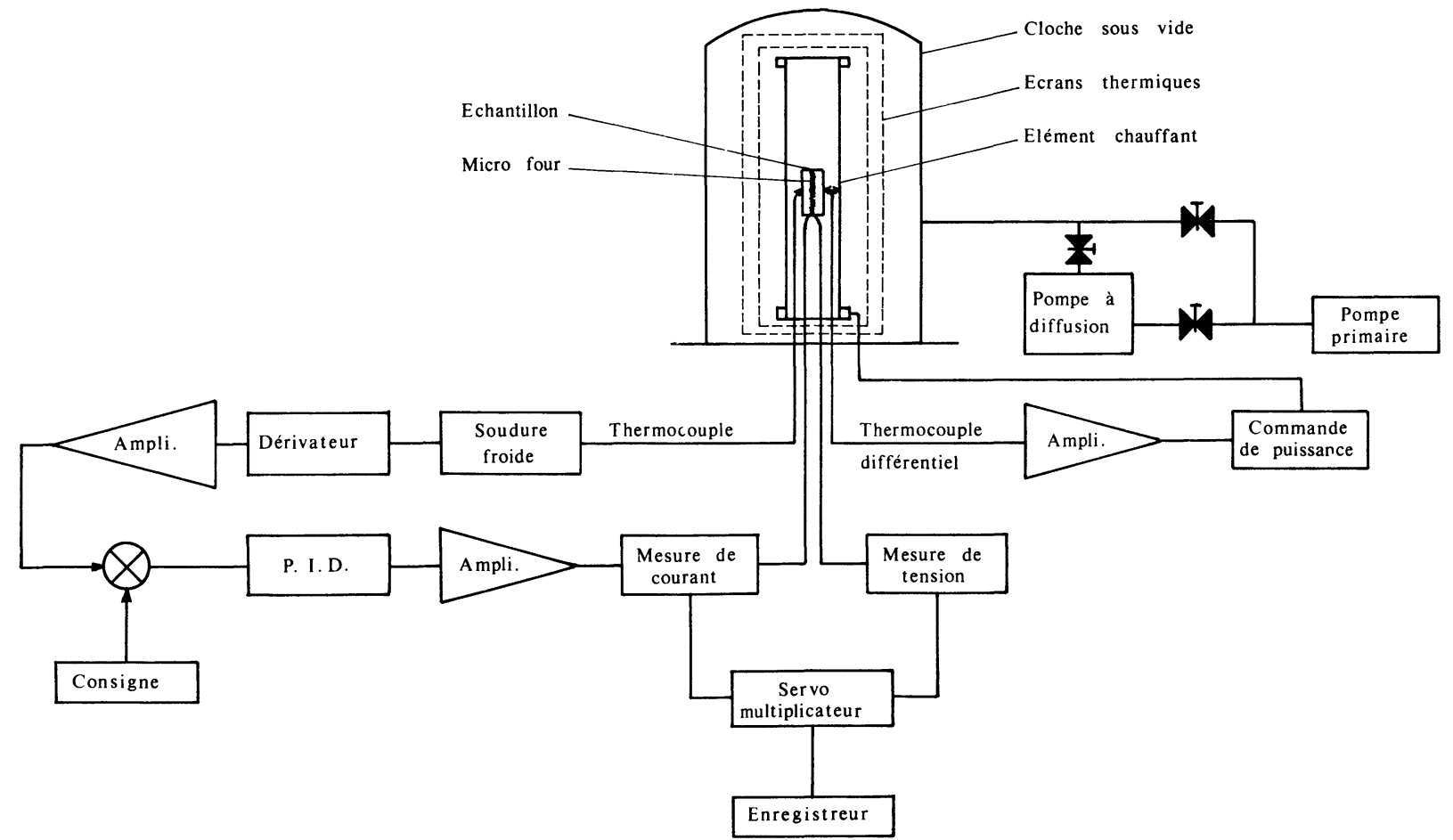

FIG. 2. - Schéma de principe de l'enthalpimètre à compensation par dérivation de la température. 


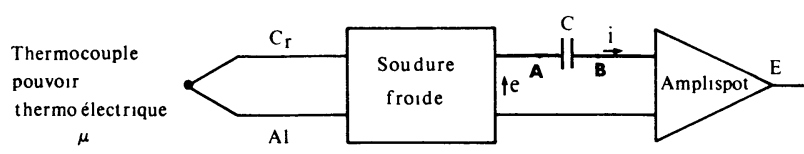

FIG. 3.

est donc proportionnelle à la dérivée de la tension du thermocouple.

L'amplificateur permet de mesurer le courant. La tension de sortie $E$ de cet amplificateur est :

$$
E=k i
$$

d'où

$$
E=k C \frac{\mathrm{d} e}{\mathrm{~d} t}
$$

$k=$ fonction de transfert de l'amplificateur.

Le dérivateur doit avoir une grande sensibilité et une grande stabilité dans le temps, afin d'obtenir des chauffages reproductibles.

La stabilité est liée à la valeur de la capacité, au gain de l'amplificateur et, surtout, à la soudure froide du thermocouple.

Afin d'augmenter la sensibilité tout en gardant une résistance de fuite élevée, de l'ordre de $3 \mathrm{M} \Omega$, nous avons choisi une capacité électrochimique au tantale de $17700 \mu \mathrm{F}$.

L'amplificateur est un amplispot de type AGC possédant une fonction de transfert tension de sortie sur courant d'entrée réglable de $10^{5}$ à $10^{8}$.

Ce type d'amplificateur galvanométrique a une impédance d'entrée très faible, $6 \Omega$ sur le calibre $10^{8}$.

La soudure froide du thermocouple doit présenter une stabilité supérieure à la résolution désirée, dans notre cas, quelques microdegrés par seconde. Cette stabilité a été obtenue en noyant la soudure froide dans un bloc de cuivre de $5 \mathrm{~kg}$ entouré de superisolant et suspendu dans une enceinte sous vide secondaire.

Cet ensemble ainsi que la capacité et l'amplificateur ont été placés dans une enceinte thermostatée à $5 / 100$ de degré près. La thermostatisation est obtenue par un ensemble chaud-froid commandé proportionnellement par une thermistance. Un ventilateur assure une bonne homogénéité de la température.

4.2 DÉTERMINATION DE LA SENSIBILITÉ DU DÉRIVATEUR. - A une température donnée, le thermocouple donne une variation de tension (de) telle que

$$
\mathrm{d} e=\mu_{\theta} \cdot \mathrm{d} \theta
$$

d'où

$$
\frac{\mathrm{d} e}{\mathrm{~d} t}=\mu_{\theta} \cdot \frac{\mathrm{d} \theta}{\mathrm{d} t}
$$

$\mu_{\theta}=$ pouvoir thermoélectrique du couple à la température $\theta$.
En reportant dans l'éq. (4)

$$
E=k C \mu_{\theta} \frac{\mathrm{d} \theta}{\mathrm{d} t} .
$$

Déterminons la vitesse minimale de montée en température de l'échantillon. Sur le calibre $10^{8}$, le bruit de fond à la sortie de l'amplificateur est inférieur à $5 \mathrm{mV} ; C=17700 \mu \mathrm{F} ; \mu=40 \mu \mathrm{V} /{ }^{\circ} \mathrm{C}$ (valeur moyenne pour un couple en chromel-alumel).

A partir de l'éq. (5), nous obtenons

$$
\frac{\mathrm{d} \theta}{\mathrm{d} t}=70 \mu^{\circ} \mathrm{C} / \mathrm{s}
$$

Si nous admettons une erreur sur la mesure de $1 / 100$, la vitesse minimale de montée en température sera de l'ordre de $25^{\circ} \mathrm{C} / \mathrm{h}$.

La vitesse maximale n'est limitée que par les temps de réponses et la puissance nécessaire pour chauffer l'échantillon. Nous avons obtenu $2500^{\circ} \mathrm{C} / \mathrm{h}$. Il semble possible d'augmenter encore cette vitesse en modifiant légèrement l'appareillage.

Les calculs précédents ne tiennent compte ni du courant de fuite de la capacité, ni de la variation du pouvoir thermoélectrique du thermocouple en fonction de la température.

Le courant de fuite $i_{\mathrm{f}}$ de la capacité peut s'écrire :

$$
i_{\mathrm{f}}=-\frac{V_{\mathrm{AB}}}{R}=\frac{e}{R}
$$

avec $R=$ résistance de fuite ; d'où le courant réel $i$ :

$$
i=C \cdot \frac{\mathrm{d} e}{\mathrm{~d} t}+\frac{e}{R}
$$

et

$$
\frac{\mathrm{d} e}{\mathrm{~d} t}=\frac{i}{C}-\frac{e}{R C}
$$

$i / C$ représente la valeur de la vitesse si le dérivateur était parfait ;

$e / R C$ représente un terme de pertes électriques uniquement fonction de $e$, c'est-à-dire de la température, mais indépendant du temps et de l'intensité choisie.

Après amplification du courant parcourant le circuit de dérivation, on obtient :

$$
E=k i=k \cdot C \cdot \frac{\mathrm{d} e}{\mathrm{~d} t}+\frac{k}{R} \cdot e .
$$

Pour que $E$ représente la dérivée exacte, il faudrait retrancher un terme égal à $k / R$.e. Les valeurs de $k$ et $R$ sont constantes, $e$ est la tension du thermocouple.

Il suffit donc de retrancher à la tension sortant de l'amplispot la tension du thermocouple amplifiée par un gain égal à $(k / R)$, d'où

$$
V=k \cdot C \cdot \frac{\mathrm{d} e}{\mathrm{~d} t}
$$

$V$ : tension représentant la vitesse de chauffage à la sortie du dérivateur. 
Il est possible de réaliser ainsi un dérivateur à très bas niveau pratiquement parfait (schéma de principe, Fig. 4). Dans notre cas, pour le calibre $10^{8}, k / R=33$, ce gain est facilement réalisable avec un amplificateur opérationnel. La linéarité du dérivateur obtenue est de l'ordre de $2 \times 10^{-3}$ pour toutes les tensions du thermocouple correspondant à la plage de température étudiée.

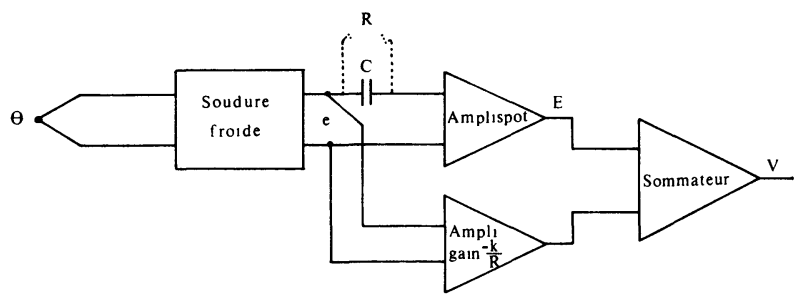

FIG. 4.

Le pouvoir thermoélectrique du chromel-alumel n'est pas constant. Il présente, en particulier, une irrégularité entre 100 et $150^{\circ} \mathrm{C}$. Il en résulte une forme ondulée de la courbe de vitesse de chauffage de l'échantillon (Fig. 5). La connaissance précise du pouvoir thermoélectrique du couple utilisé permet d'apporter une correction si l'on désire déterminer exactement la vitesse de chauffage à chaque température.

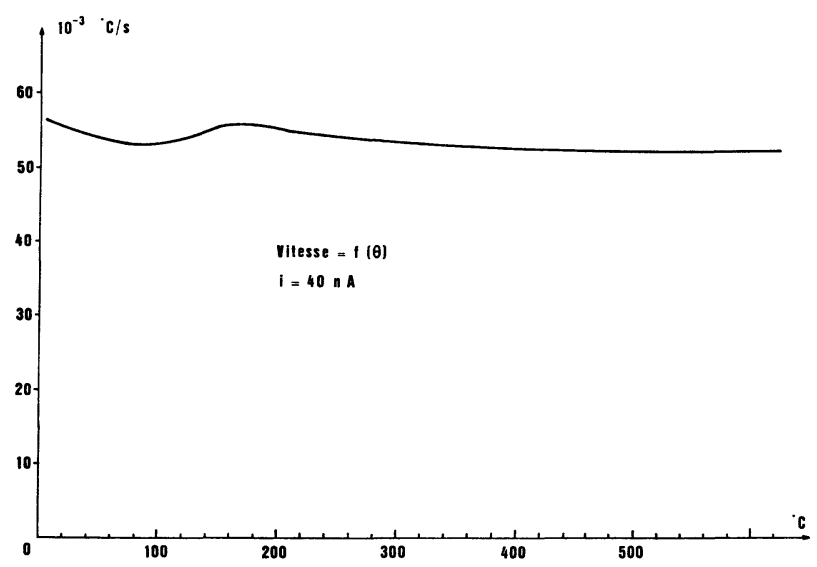

FIG. 5. - Vitesse de chauffage obtenue en fonction de la température pour une intensité donnée.

Ce dérivateur de température n'est donc pas parfait, mais il possède une grande sensibilité qui permet de détecter de très faibles variations de pente, de l'ordre de $40 \mu^{\circ} \mathrm{C} / \mathrm{s}$. Les causes de non-linéarité sont purement physiques et constantes, la reproductibilité d'un chauffage à l'autre est voisine de 1/1000.

Ce dérivateur nous a donné pleine satisfaction, surtout par sa fidélité, sa sensibilité et sa simplicité d'utilisation.

5. Réalisation de l'ensemble. - 5.1 L'ÉCHANTILLON est cylindrique, de 6 à $12 \mathrm{~mm}$ de diamètre et de $14 \mathrm{~mm}$ de longueur environ. Il comporte trois logements (Fig. 6).

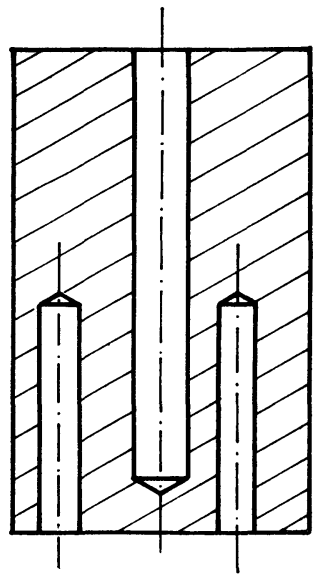

Fig. 6. - Coupe d'un échantillon massif.

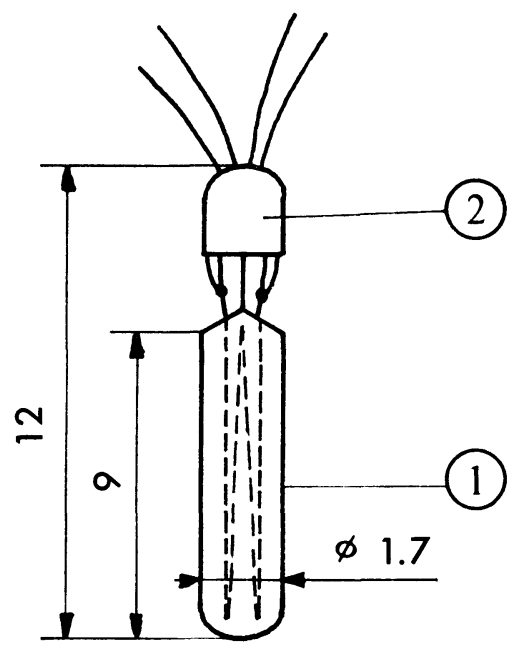

FIG. 7. - Microfour.

Le trou central reçoit l'élément chauffant (microfour). Les deux autres logements reçoivent les thermocouples.

L'échantillon peut être massif pour l'étude des corps solides. Pour les poudres, il peut être remplacé par une nacelle.

5.2 LES THERMOCOUPLES sont des thermocoax en chromel-alumel de $1 \mathrm{~mm}$ de diamètre. Celui qui est placé le plus à l'extérieur est monté en différentiel avec le thermocouple mesurant la température de l'enceinte. L'autre thermocouple sert à mesurer la vitesse de chauffage.

5.3 LA CONSIGNe. - Elle permet de déterminer le courant $i$ que l'on veut faire circuler dans le dérivateur, c'est-à-dire la vitesse de chauffage. Elle est constituée par une alimentation stabilisée, à double diode Zener, qui délivre une tension réglable mise en opposition avec la tension du dérivateur. Sa stabilité doit être au moins égale à $10^{-4}$.

5.4 LE RÉGulateur. - Nous avons utilisé jusqu'à présent un régulateur PID qui s'est avéré très 
souple lors de la mise au point de notre installation. Dans une version définitive, il semblerait qu'un amplificateur de puissance donnerait des résultats satisfaisants.

Le régulateur délivre une tension qui est fonction de l'écart entre la tension du dérivateur et celle de la consigne. Cette tension est appliquée aux bornes du microfour noyé dans l'échantillon.

La mesure du courant parcourant cette résistance et de la tension à ses bornes permet de déterminer la puissance électrique utilisée pour chauffer l'échantillon.

5.5 LA RÉSISTANCE CHAUFFANTE OU " MICROFOUR » est une résistance en platine rhodié à $5 \Omega$, logée dans le trou central de l'échantillon (Fig. 7). Elle comprend quatre fils de sortie.

Le support (1) est en alumine de diamètre extérieur $1,7 \mathrm{~mm}$. L'écran thermique (2) sert de support aux fils d'amenées.

Deux des fils conduisent le courant, les deux autres permettent de mesurer la tension aux bornes de la résistance.

5.6 RÉAlisation DE L'enceinte adiabatiQue. L'échantillon doit être placé dans des conditions les plus adiabatiques possibles. Nous avons choisi l'utilisation d'un four sous vide secondaire à parois froides.

L'élément chauffant forme directement la paroi intérieure de l'enceinte adiabatique. Il est constitué par un tube en graphite de grande longueur, par rapport aux dimensions de la pièce, pour diminuer les pertes par rayonnement. Le diamètre du tube est de $35 \mathrm{~mm}$ et sa longueur de $400 \mathrm{~mm}$. Il est entouré extérieurement par des écrans réfléchissants. La puissance disponible est de $3 \mathrm{~kW}$, ce qui permet d'atteindre une température de $1000{ }^{\circ} \mathrm{C}$ en moins de $5 \mathrm{mn}$. Ce four présente une inertie très faible, d'où des temps de réponse courts, de l'ordre de 4 à $5 \mathrm{~s}$.

Ce tube en graphite, chauffé par effet Joule, est asservi à suivre, à chaque instant, la température de l'échantillon qu'il contient. Un thermocouple, monté en différentiel entre la paroi interne du tube de graphite et l'échantillon, commande, par l'intermédiaire d'un régulateur, la puissance à fournir à ce tube pour le chauffer à la même température que l'échantillon. L'échantillon est positionné dans l'axe du four à mihauteur par les fils du "microfour» et des thermocouples.

Une telle enceinte n'est pas parfaitement adiabatique. Bien que le four suive à mieux de un dixième de degré la température de l'échantillon, de faibles pertes thermiques subsistent : pertes par rayonnement vers les extrémités du tube, pertes par conduction par les thermocouples et les fils du microfour.

Néanmoins, pour deux chauffages, dans des conditions identiques, la reproductibilité des pertes demeure parfaite. On peut donc penser que ces pertes, pour une vitesse de chauffage donnée, ne sont fonction que de la température.
6. Etude des courbes de puissance. - Dans le cas d'une enceinte quasi adiabatique, l'expression (1), qui donne la puissance nécessaire au chauffage, peut s'écrire :

$$
P=K m C_{\theta} V_{\theta}+P_{\theta}
$$

$P_{\theta}=$ pertes thermiques à la température $\theta$.

A partir de l'éq. (9), on déduit l'expression de $V_{\theta}$ :

$$
V_{\theta}=\frac{\mathrm{d} \theta}{\mathrm{d} t}=\frac{1}{\mu_{\theta}} \frac{\mathrm{d} e}{\mathrm{~d} t}=\frac{V}{k C \mu_{\theta}}
$$

d'où

$$
P=\frac{K m C_{\theta}}{\mu_{\theta} k C} V+P_{\theta} .
$$

Pour une valeur de $V$ donnée, on peut tracer la courbe représentant le premier terme de l'éq. (11) en fonction de la température $\theta$.

La comparaison avec une courbe expérimentale permet d'apprécier les pertes $P_{\theta}$ (Fig. 8).

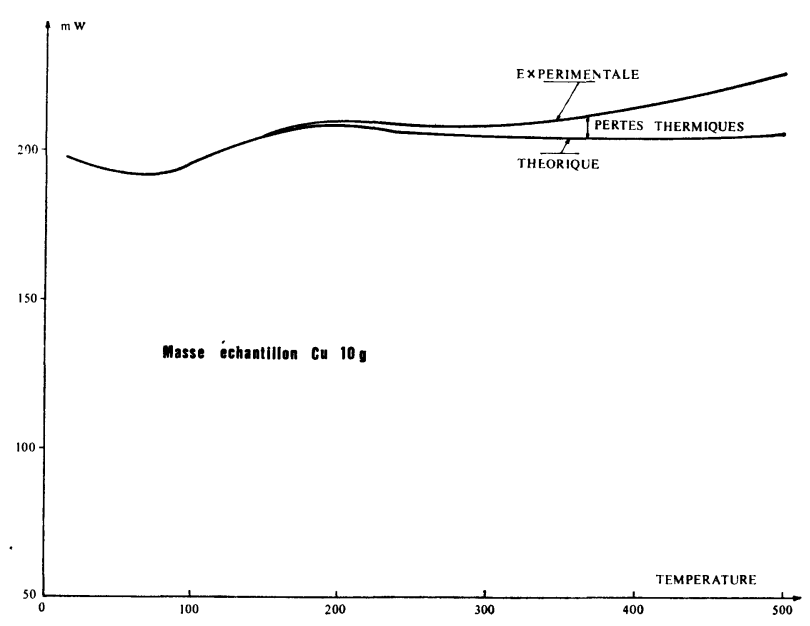

Fig. 8. - Puissance nécessaire au chauffage d'un échantillon en cuivre de $10 \mathrm{~g}$ en fonction de la température. Courbes expérimentale et théorique.

La courbe représentée est relative à une pièce de cuivre de $10 \mathrm{~g}$ chauffée à une vitesse de $150^{\circ} \mathrm{C} / \mathrm{h}$.

La cassure observée vers $170^{\circ} \mathrm{C}$ est due à la variation du pouvoir thermoélectrique du couple chromelalumel.

Les pertes croissent avec la température, mais elles ne dépassent pas $20 \%$ de la puissance nécessaire au chauffage de la pièce. Nous verrons ultérieurement qu'elles n'interviennent pas dans la détermination des énergies de transformations.

7. Causes d'erreurs possibles sur la mesure des énergies. - 7.1 CAUSES DUES A LA MESURE (inexactitude ou non-reproductibilité). - 7.1.1 Mesure de la dérivée. - La valeur absolue exacte de la dérivée est peu importante. Par contre, la fidélité et la linéarité de la dérivée sont essentielles. Ces caractéristiques sont assurées à $0,2 \%$ près. La résolution observée expéri- 
mentalement est de $40 \mu^{\circ} \mathrm{C} / \mathrm{s}$, la stabilité est supérieure à $70 \mu^{\circ} \mathrm{C} / \mathrm{s}$ sur $48 \mathrm{~h}$.

7.1.2 Mesure de puissance. - Elle est effectuée actuellement par un servomultiplicateur couplé à un enregistreur. La précision de cet ensemble est assurée à $1 \%$ près.

7.1.3 Mesure des énergies. - L'énergie d'une transformation est directement proportionnelle à l'aire du pic observé sur la courbe de puissance. Il faut donc interpoler la ligne de base. La précision sur la mesure de la surface sera d'autant meilleure que celle-ci sera plus importante et que la distance interpolée sera plus réduite.

L'amplification de la mesure de puissance est limitée par le bruit de fond. Celui-ci représente approximativement $0,1 \%$ de la puissance nécessaire au chauffage. Un bruit de fond de $1 \mathrm{~mm}$ (tolérable sur l'enregistrement) entraîne une amplitude de $1 \mathrm{~m}$, ce qui oblige à stocker trois ou quatre échelles pour garder cette sensibilité sur l'enregistreur.

La vitesse de défilement du papier définit l'échelle des temps et permet d'étaler plus ou moins les pics de transformations. Il est possible d'obtenir ainsi la même sensibilité pour différentes vitesses de chauffage comme le montre le tableau ci-dessous.

\begin{tabular}{|c|c|c|c|}
\hline $\begin{array}{l}\text { Vitesse } \\
\text { de } \\
\text { chauffage }\end{array}$ & $\begin{array}{l}\text { Puissance } \\
\text { à la pleine } \\
\text { échelle }\end{array}$ & $\begin{array}{l}\text { Vitesse de } \\
\text { défilement } \\
\text { du papier }\end{array}$ & $\begin{array}{c}\text { Energie } \\
\text { par unité } \\
\text { de surface }\end{array}$ \\
\hline- & - & $\mathrm{F}=\mathrm{T}$ & 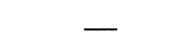 \\
\hline $25^{\circ} \mathrm{C} / \mathrm{h}$ & $10 \mathrm{~mW}$ & $1,2 \mathrm{~mm} / \mathrm{mn}$ & $200 \mathrm{~mJ} / \mathrm{cm}^{2}$ \\
\hline $100^{\circ} \mathrm{C} / \mathrm{h}$ & $25 \mathrm{~mW}$ & $3 \mathrm{~mm} / \mathrm{mn}$ & $200 \mathrm{~mJ} / \mathrm{cm}^{2}$ \\
\hline $200^{\circ} \mathrm{C} / \mathrm{h}$ & $50 \mathrm{~mW}$ & $\mathrm{~mm} / \mathrm{mn}$ & $200 \mathrm{~mJ} / \mathrm{cm}^{2}$ \\
\hline $1000^{\circ} \mathrm{C} / \mathrm{h}$ & $250 \mathrm{~mW}$ & $\mathrm{~mm} / \mathrm{mn}$ & $200 \mathrm{~mJ} / \mathrm{cm}^{2}$ \\
\hline $2000^{\circ} \mathrm{C} / \mathrm{h}$ & $500 \mathrm{~mW}$ & $\mathrm{~mm} / \mathrm{mn}$ & $200 \mathrm{~mJ} / \mathrm{cm}^{2}$ \\
\hline
\end{tabular}

Dans ce cas, pour un échantillon de $10 \mathrm{~g}$, la sensibilité est de $20 \mathrm{~mJ} / \mathrm{g} / \mathrm{cm}^{2}$.

La mesure d'énergie présente donc une erreur relative systématique due à la mesure de la puissance, soit $1 \%$, plus une erreur absolue qui dépend de la détermination de l'aire du pic. Cette erreur peut avoir deux causes :

- La précision de la planimétration. Pour un manipulateur expérimenté, l'erreur est pratiquement indépendante de la surface et peut être chiffrée à $\pm 0,08 \mathrm{~cm}^{2}$. Cette erreur devient donc négligeable pour des surfaces supérieures à $10 \mathrm{~cm}^{2}$.

- L'interpolation de la ligne de base. L'erreur ainsi commise peut être considérée comme étant égale à l'aire obtenue en multipliant le bruit de fond observé par la longueur de la ligne de base interpolée.

Pour une surface supérieure à $10 \mathrm{~cm}^{2}$, on peut donc écrire :

$$
\begin{aligned}
& \text { Energie }=(\text { aire } \times \text { sensib. }) \pm \\
& \pm\left[\frac{\text { long. interpolée } \times \text { bruit de fond } \times 100}{\text { aire }}+1\right] \% .
\end{aligned}
$$

7.2 CAUSES THERMIQUeS. - 7.2.1 Enceinte adiabatique. - L'exactitude de la mesure de la puissance dépend des pertes thermiques, cf. éq. (11). Les puissances de réactions apparaissent comme un terme supplémentaire dans cette équation

$$
P=\frac{K m C_{\theta}}{\mu_{\theta} k C} \cdot V+P_{\theta}-Q_{\theta}
$$

$Q_{\theta}$ : puissance dégagée par la réaction à la température $\theta$.

Les pertes thermiques provoquent simplement une dérive de la ligne de base, mais n'affectent pas la mesure des énergies de transformations. Cette dérive peut rendre l'interpolation de la ligne de base plus délicate et obliger à réduire la sensibilité.

L'enceinte doit donc être aussi adiabatique que possible. La réalisation particulièrement soignée de l'élément chauffant (forme, matériau et usinage) a permis d'obtenir une bonne homogénéité de la température, d'où de faibles pertes thermiques. Elles ne dépassent pas $20 \%$ de la puissance nécessaire au chauffage de l'échantillon dans le cas de montées en températures lentes. Elles ne sont pratiquement fonction que de la température et deviennent donc négligeables pour les chauffages rapides.

7.2.2 Gradient de température dans l'échantillon. - La forme cylindrique et le chauffage coaxial d'un échantillon de petites dimensions (6 à $12 \mathrm{~mm}$ de diamètre et $14 \mathrm{~mm}$ de longueur) constituent des conditions favorables à l'obtention d'un faible gradient. Un calcul a donné $0,05^{\circ} \mathrm{C}$ d'écart entre le cœur et la périphérie d'un échantillon de cuivre de $10 \mathrm{~mm}$ de diamètre chauffé à $1000^{\circ} \mathrm{C} / \mathrm{h}$. On peut considérer que tout l'échantillon est à la même température.

7.2.3 Couplage thermique microfour-échantillon. Il est indispensable de vérifier que toute l'énergie apportée par le microfour est communiquée à l'échantillon.

Pour ce faire, un deuxième microfour a été noyé dans un échantillon de cuivre, afin de simuler des réactions d'énergies bien déterminées. La puissance apportée par ce deuxième microfour doit provoquer une diminution, d'une quantité égale, de la puissance nécessaire au chauffage de l'échantillon quelles que soient la température et la vitesse de chauffage.

Nous présentons, figure 9 , quelques résultats extraits d'une étude systématique.

Nous observons une bonne correspondance entre la puissance envoyée au microfour supplémentaire et la diminution de puisance observée.

L'erreur introduite par le couplage semble négligeable par rapport à l'erreur sur la détermination de l'énergie.

7.3 CAUSES ÉLECTRIQUES. - Lors de transformations rapides, le temps de réponse du système asservi ne permet pas de compenser à chaque instant le phénomène. 

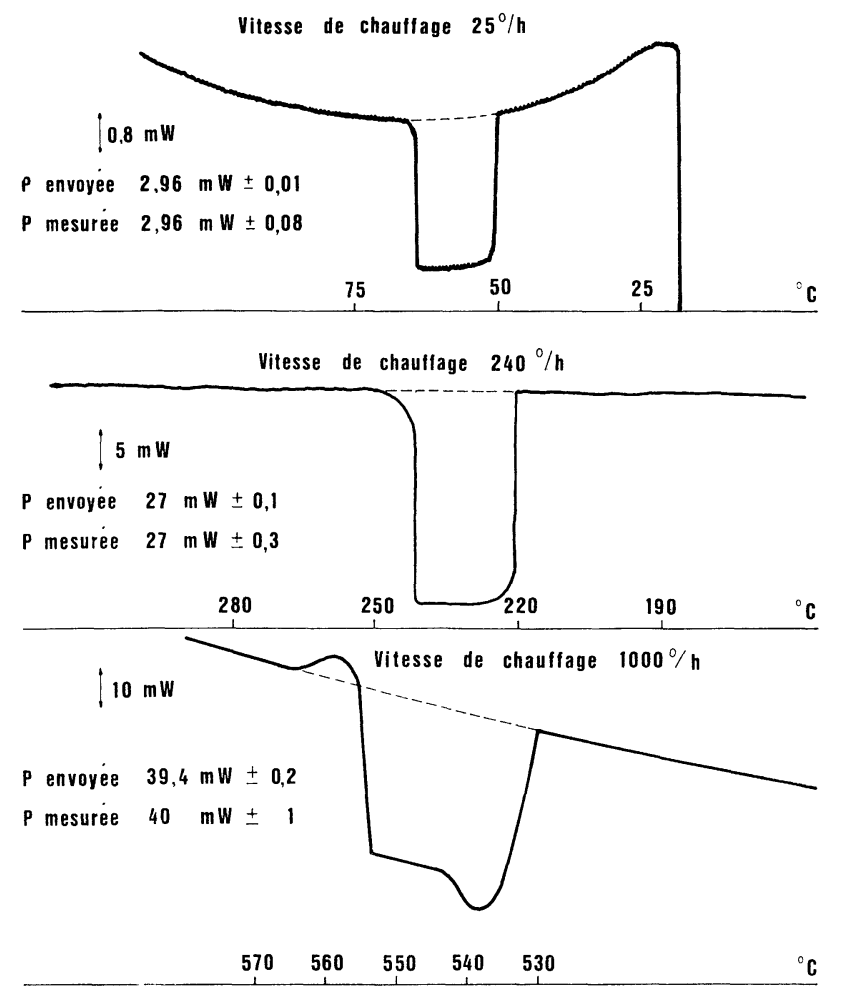

FIG. 9. - Réponse du système à une entrée de puissance en créneau pour différentes vitesses de chauffage et différentes températures.

Afin de juger de l'erreur qui en résulte, nous avons, grâce au-montage à deux microfours décrit précédem- ment, provoqué des pics (créneaux) d'énergie constante, mais de formes différentes, figure 10.

Il semble que, même dans le cas de pics brefs, l'énergie apportée soit entièrement compensée.

Ce résultat montre également que l'échantillon est bien dans des conditions quasi adiabatiques puisqu'il n'y a pas de perte d'énergie.

A la limite, le temps de réponse du système intervient peu sur la mesure des énergies de réaction.

Par contre, la détermination de la cinétique d'une réaction nécessite une compensation instantanée et rigoureuse. Dans ce cas, on contrôle l'exactitude de la pente de chauffage lors de la réaction. Un choix judicieux des constantes du programmateur permet d'améliorer considérablement les temps de réponses au voisinage d'une température donnée. On a pu ainsi mesurer des vitesses de réaction de $30 \mathrm{~mW} / \mathrm{s}$ avec une erreur sur la pente inférieure à $1 \%$.

7.4 ConClusion. - Notre appareillage permet de compenser complètement les transformations dans pratiquement tous les cas où des réactions mettent en jeu des énergies faibles. Si la puissance de la réaction est trop importante, il y a lieu de prendre une masse de matière plus faible et de l'insérer dans un échantillon en cuivre.

Les causes principales d'erreur résident dans la mesure de puissance $(1 \%)$ et dans la détermination de l'aire correspondant aux transformations. Nous donnons quelques exemples d'application dans le chapitre suivant.

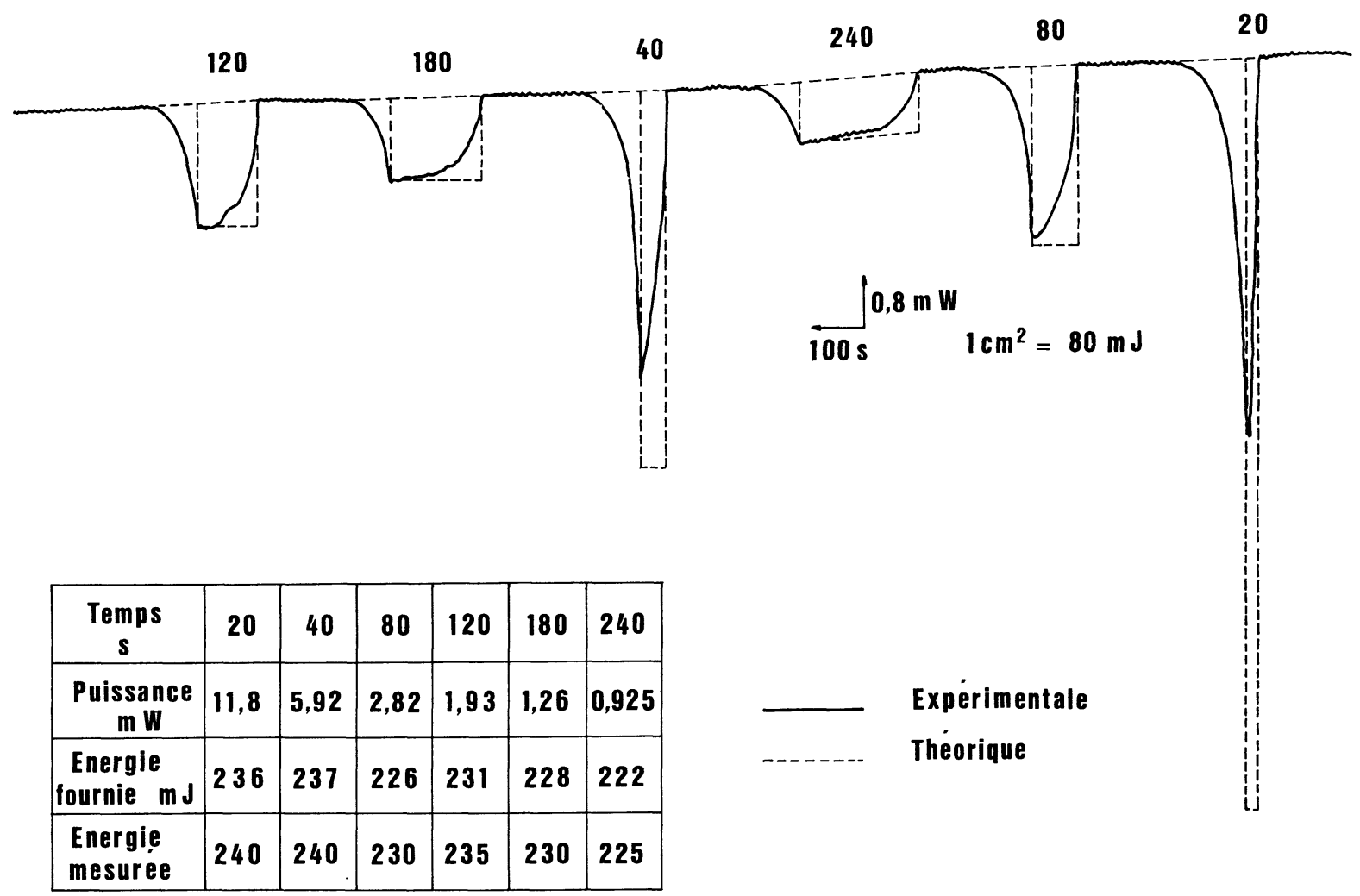

Fig. 10. - Réponse du système à des entrées en créneaux de même énergie, mais de formes différentes. 
8. Exemples d'application. - Nous présentons, à titre d'exemple d'application, une partie d'une étude sur l'hystérésis de la transformation du cobalt en fonction de la vitesse de chauffage.

La transformation du cobalt a déjà fait l'objet de nombreux travaux [6]. Le cobalt présente, vers $417^{\circ} \mathrm{C}$, une transformation allotropique endothermique au chauffage avec passage d'une structure hexagonale compacte $\varepsilon$ à une structure cubique à faces centrées $\alpha$. D'après certains auteurs [7], cette transformation présente une hystérésis faible pour les vitesses de chauffage inférieures à $200^{\circ} \mathrm{C} / \mathrm{h}$. Une détermination de l'hystérésis pour des vitesses beaucoup plus élevées nous a paru intéressante.

L'énergie de transformation au chauffage pour un polycristal est de $108,7 \mathrm{cal} / \mathrm{mole}$ à $1 \%$ près [8]. Cette énergie importante ne nous a pas permis d'effectuer des essais sur un échantillon massif. Cinq petits barreaux de $2 \mathrm{~mm}$ de diamètre et de $12 \mathrm{~mm}$ de longueur d'un cobalt industriel à $99,9 \%$ ont été noyés dans un échantillon de cuivre de $10 \mathrm{~mm}$ de diamètre. La masse de cobalt est alors de 1,833 g. Elle doit dégager une énergie de transformation de 14,2 J. La masse totale de l'échantillon est de $9,42 \mathrm{~g}$, ce qui représenterait une énergie de $1,5 \mathrm{~J} / \mathrm{g}$, si cette réaction était rapportée à toute la masse de l'échantillon.

Nous avons utilisé les vitesses de chauffage suivantes : $50^{\circ} \mathrm{C} / \mathrm{h}, \quad 100^{\circ} \mathrm{C} / \mathrm{h}, \quad 200^{\circ} \mathrm{C} / \mathrm{h}, \quad 500^{\circ} \mathrm{C} / \mathrm{h}$, $750^{\circ} \mathrm{C} / \mathrm{h}, 1000^{\circ} \mathrm{C} / \mathrm{h}, 2000^{\circ} \mathrm{C} / \mathrm{h}$. Un choix judicieux des sensibilités et des vitesses de défilement a permis d'obtenir la même sensibilité pour tous ces chauffages, soit : $0,2 \mathrm{~J} / \mathrm{cm}^{2}$.

Ces vitesses de chauffage élevées permettent un gain de temps important, car il est possible d'effectuer jusqu'à 4 montées en température par jour.

La courbe, figure 11, a été obtenue pour un chauffage à $750^{\circ} \mathrm{C} / \mathrm{h}$ : la surface du pic est de $72 \mathrm{~cm}^{2}$, la longueur interpolée : $15 \mathrm{~cm}$, le bruit de fond est de l'ordre de $\pm 0,5 \mathrm{~mm}$.

Surface correspondant à l'erreur possible :

$$
15(2 \times 0,5)=1,5 \mathrm{~cm}^{2}
$$

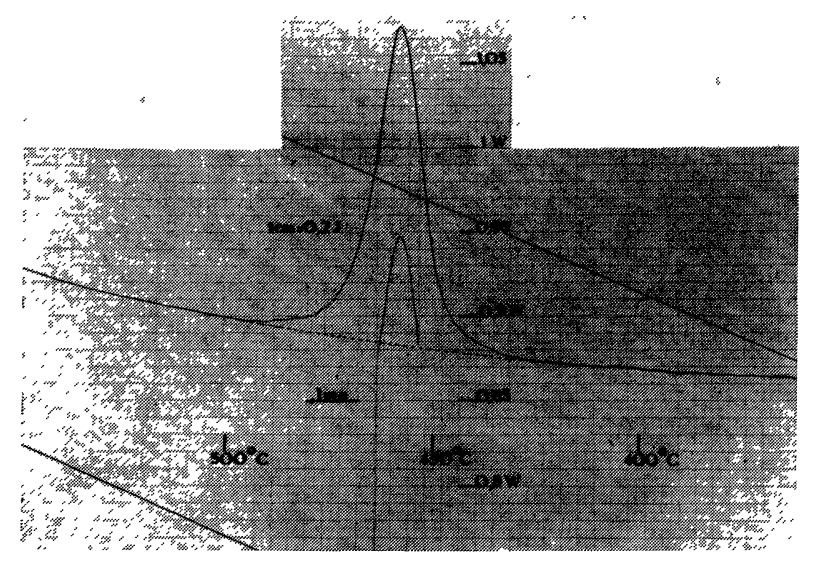

Fig. 11. - Photographie d'une courbe expérimentale sur la transformation allotropique du cobalt au chauffage. d'où l'énergie absorbée :

$$
\begin{aligned}
W & =(72 \times 0,2) \pm \frac{1,5 \times 100}{72}+1 \\
& =14,4 \mathrm{~J} \text { à } 3 \% \text { près } .
\end{aligned}
$$

Nous avons porté les résultats obtenus sur la figure 12, la courbe du haut représente les énergies absorbées en fonction de la vitesse de chauffage. On remarque très nettement l'allure croissante de la courbe. L'énergie absorbée semble donc croître avec la vitesse de chauffage. L'hystérésis est de l'ordre de $15 \%$ pour cette gamme de vitesses, ce qui semble effectivement faible, surtout pour les chauffages lents. L'énergie tend alors vers $14 \mathrm{~J}$, soit $110 \mathrm{cal} / \mathrm{mole}$ à $3 \%$ près.

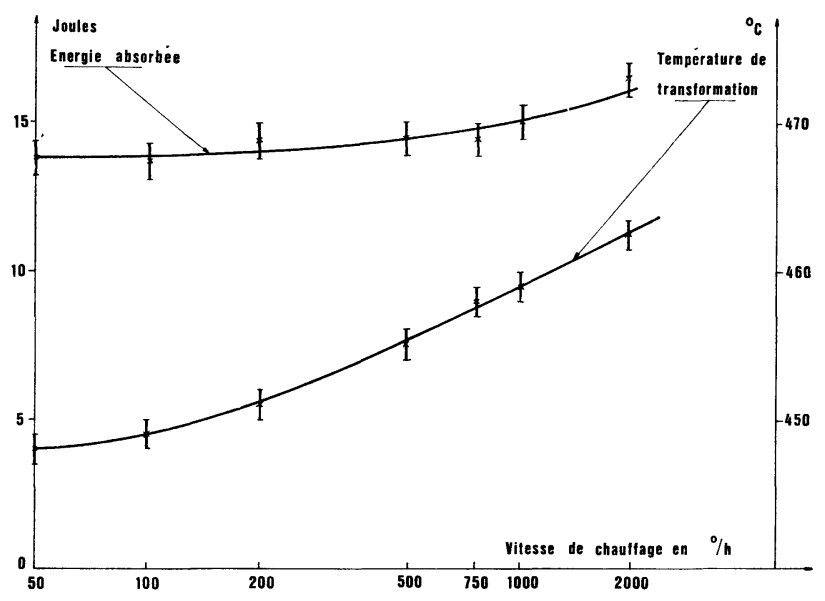

Fig. 12. - Hystérésis observée sur l'énergie absorbée et la température du maximum de réaction en fonction de la vitesse de chauffage.

La courbe du bas représente la température du maximum de la réaction. Celle-ci peut être déterminée avec précision $\left( \pm 1^{\circ} \mathrm{C}\right)$, car le pic est très pointu. La température du maximum de transformation croît avec la vitesse de chauffage. L'hystérésis est de $14^{\circ} \mathrm{C}$ pour des vitesses variant dans un rapport de 1 à 40 .

Si nous rapportions ce pic de $72 \mathrm{~cm}^{2}$ à la masse totale de l'échantillon (cuivre + cobalt), cette transformation équivaudrait à une absorption d'énergie de $0,37 \mathrm{cal} / \mathrm{g}$.

Notre appareillage permet également, de par sa bonne reproductibilité, la détermination précise de l'énergie des réactions irréversibles se déroulant sur des intervalles de température importants. La ligne de base est obtenue par un deuxième chauffage dans des conditions rigoureusement identiques. Nous avons étudié ainsi le revenu d'un acier XC 52 trempé à l'eau. Celui-ci présente plusieurs réactions irréversibles se déroulant entre l'ambiante et $600^{\circ} \mathrm{C}$ (9), la courbe (13) représente la différence de puissance observée entre la première et la deuxième montée en température. 


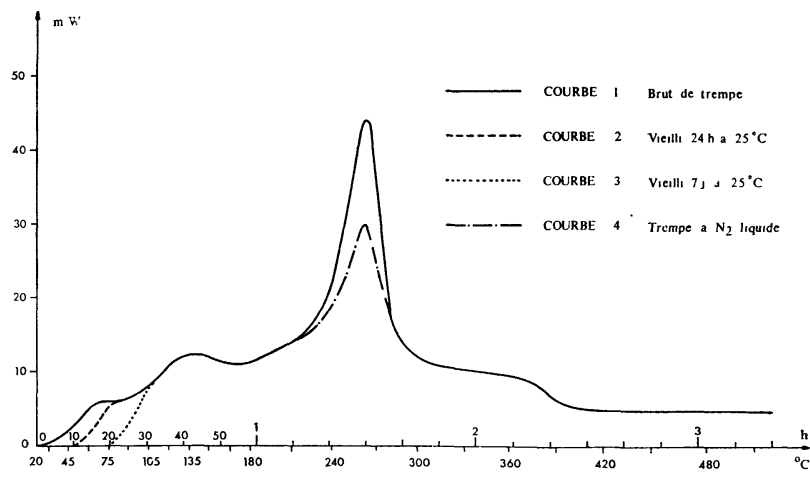

FIG. 13. - Puissance dégagée lors du revenu après trempe d'un échantillon d'acier à $0,52 \%$ de carbone en fonction du temps et de la température.

9. Conclusion. - Notre appareillage permet des mesures directement quantitatives des énergies et des cinétiques de réactions entre l'ambiante et $900^{\circ} \mathrm{C}$. Il ne nécessite qu'un seul échantillon. La bonne repro- ductibilité d'un essai à l'autre permet également une détermination approchée en continu des chaleurs massiques en utilisant une faible quantité de matière. Le travail sous vide permet d'éviter toute oxydation.

La précision sur la mesure des énergies est de l'ordre de $3 \%$ pour des réactions qui mettent en jeu des énergies supérieures à $0,1 \mathrm{~J} / \mathrm{g}$ sur un intervalle de température de $50^{\circ} \mathrm{C}$. La précision diminue pour des énergies plus faibles. Elle n'est plus que de $5 \%$, par exemple, pour $0,05 \mathrm{~J} / \mathrm{g}$.

Nous employons cet appareil pour des études métallurgiques, mais son utilisation peut être étendue à toutes les disciplines ayant recours à l'analyse thermique.

Il apporte, de plus, une gamme très étendue de vitesses de chauffage de $25^{\circ} \mathrm{C} / \mathrm{h}$ à $2500^{\circ} \mathrm{C} / \mathrm{h}$, une simplification de l'appareillage, tout en conservant une sensibilité et une précision comparables, sinon supérieures, à celles des autres appareils d'analyse thermique quantitative à compensation.

\section{Bibliographie}

[1] Thermal Analysis' 65, Proceedings of the First International Congress at Aberdeen (J. P. Redfern).

[2] Eyraud, L., Diélectriques solides anisotropes et ferroélectricité (Gauthier-Villars, Paris) 1967.

[3] Bonjour, E., Calorimètre différentiel à puissance compensée CPC, Brevet no 1.363.283 du 26.3.1963.

[4] Fetiveau, Y., Thèse de Doctorat ès Sciences Physiques, Lyon (1969).
[5] SEFRAM, «Analyse thermique dérivée », NT 102.

[6] Hultgren, Select. Values of Termo. Prop. of Metals., Univ. of Calif., Berkley.

[7] Jeanjean, R., Dubois, J., Fetiveau, Y., Rivière, R., Mémoires Scientifiques Rev. Métallurg.

[8] Adams, R., Alstetler, C., Trans. AIME 242 (1968) 139.

[9] Bigot, R., Cercle d'Etude des Métaux (1968) X 12553. 